\title{
Electronic Structure of $\mathrm{BiFe}_{1-x} \mathrm{Mn}_{\mathbf{x}} \mathrm{O}_{3}$ Thin Films Investigated by X-Ray Absorption Spectroscopy
}

\author{
Abduleziz Ablat, ${ }^{1,2}$ Emin Muhemmed, ${ }^{1}$ Cheng Si, ${ }^{1}$ Jiaou Wang, ${ }^{1}$ Haijie Qian, ${ }^{1}$ Rui Wu, \\ Nian Zhang, ${ }^{1}$ Rong Wu, ${ }^{2}$ and Kurash Ibrahim ${ }^{1}$ \\ ${ }^{1}$ Beijing Synchrotron Radiation Facility, Institute of High Energy Physics, CAS, Beijing 100049, China \\ ${ }^{2}$ School of Physical Science and Technology, Xinjiang University, Urumqi 830046, China \\ Correspondence should be addressed to Kurash Ibrahim, kurash@ihep.ac.cn
}

Received 7 March 2012; Accepted 21 April 2012

Academic Editor: Weichang Hao

Copyright (C) 2012 Abduleziz Ablat et al. This is an open access article distributed under the Creative Commons Attribution License, which permits unrestricted use, distribution, and reproduction in any medium, provided the original work is properly cited.

\begin{abstract}
Multiferroic polycrystalline $\mathrm{BiFe}_{1-x} \mathrm{Mn}_{x} \mathrm{O}_{3}(0 \leq x \leq 0.3)$ thin films have been prepared on the $\mathrm{Pt}(111) / \mathrm{Ti} / \mathrm{SiO} \mathrm{O}_{2} / \mathrm{Si}(100)$ substrates by pulsed laser deposition method. The influence of $\mathrm{Mn}$ substitution on the electronic structure and magnetic properties has been studied. X-ray diffraction spectroscopy shows that $\mathrm{Mn}$ substitution slightly modulates crystal structure of the $\mathrm{BiFe}_{1-x} \mathrm{Mn}_{x} \mathrm{O}_{3}$ system within the same structural phase. According to Fe $L$ edge X ray absorption spectroscopy, Fe ions are found to be formally trivalent for doping amount $x$ in $\mathrm{BiFe}_{1-x} \mathrm{Mn}_{x} \mathrm{O}_{3}$. The enhanced magnetization by increasing $\mathrm{Mn}$ content is attributed to an alternation degree of hybridization between Fe $3 d-\mathrm{O} 2 p$ and $\mathrm{Mn} 3 d-\mathrm{O} 2 p$ orbitals, basing on the carefully examined Fe $L$ and $\mathrm{O} K$ edge X-ray absorption spectroscopy. The crystal structural and the electronic structural results show a causal relation between them by demonstrating intrinsic mutual dependence between respective variations.
\end{abstract}

\section{Introduction}

Multiferroics are a group of materials which simultaneously show up several kinds of ferroic properties such as ferroelectricity, ferromagnetism, and ferroelasticity in the same phase [1]. In ferromagnetic-ferroelectric multiferroics, magnetization can be tuned by an applied electric field and electronic polarization by magnetic field [2]. The magnetoelectric (ME) effects of multiferroics imply important technological application perspectives in multifunctional devices like high-temperature transistor gate, memory device, and spintronics. Few multiferroics exhibits as natural occurring phases, examples of which include $\mathrm{YMnO}_{3}, \mathrm{PbVO}_{3}, \mathrm{BiCrO}_{3}$, $\mathrm{BiMnO}_{3}$ and $\mathrm{BiFeO}_{3}$ (BFO) $[3,4]$. Among them only the $\mathrm{BFO}$ exhibits ME effects at room temperature. Although the $\mathrm{BFO}$ is considered to be a room-temperature multiferroics, its ME effect is too low because of weak ferromagnetism. Two different approaches have been used for enhancing the ME effect in BFO. One is to bring the ferroelectric $T_{C}$ or ferromagnetic $T_{N}$ transition temperature to around room temperature by cationic substitutions [5]. The other is first to achieve phase transition from antiferromagnetic to ferromagnetic and then increase magnetic properties while retaining the ferroelectricity through strain modification [6, 7]. Among these, Mn doping is the most common method to achieve an enhanced magnetization. This is because the $\mathrm{BiMnO}_{3}-\mathrm{a} \mathrm{Mn}$ centered compound of $\mathrm{BiMO}_{3}$ series-exhibits ferromagnetism below $\sim 105 \mathrm{~K}$ and ferroelectric at $\sim 450 \mathrm{~K}[8]$. The $\mathrm{BiMnO}_{3}$ has a large ferromagnetic magnetization, implying that the Mn doping in $\mathrm{BFO}$ improves the magnetization and enhances the ME effects more effectively than other element substitution. Mechanisms for the increasing magnetization in Mn-doped BFO systems have been discussed in terms of canting of the antiferromagnetically ordered spins by structural distortion [9], double exchange interaction induced by hole doping effects [10], and variation of $\mathrm{Fe}^{3+}$ ion concentration [11]. Investigation in terms of electronic structure is a direct way to understand the macroscopic properties of materials, due to intrinsic relationship between the electronic structure and macroscopic properties. 
In this work, we prepared Mn-substituted $\mathrm{BiFe}_{1-x} \mathrm{Mn}_{x} \mathrm{O}_{3}$ (BFMO) thin films by pulsed laser deposition (PLD). The crystal structure, surface morphology, magnetic properties, and electronic structure of the Mn-doped BFMO systems were investigated via X-ray diffraction (XRD), scanning electron microscopy (SEM), Alternating Gradient force Magnetometer (AGM), and X-ray absorption spectroscopy (XAS). Origin for the enhanced magnetization of the BFMO system with increased Mn amount of doping is investigated in terms of their electronic structural changes.

\section{Experiment}

The $\mathrm{BiFe}_{1-x} \mathrm{Mn}_{x} \mathrm{O}_{3}(0 \leq x \leq 0.3)$ films were prepared by $\mathrm{PLD}$ method on the $\mathrm{Pt}(111) / \mathrm{Ti} / \mathrm{SiO}_{2} / \mathrm{Si}(100)$ substrates in a PLD chamber connected to the photoemission spectroscopy (PES) system at 4B9B beam line of the Beijing Synchrotron Radiation Facility. Ceramic BFMO targets of $20 \mathrm{~mm}$ diameter $2 \mathrm{~mm}$ thick for PLD were prepared by sintering the mixtures of $\mathrm{Bi}_{2} \mathrm{O}_{3}$ (99.99 at.\%), $\mathrm{Fe}_{2} \mathrm{O}_{3}$ (99.99 at.\%), and $\mathrm{Mn}_{2} \mathrm{O}_{3}$ (99.99 at.\%) at $820^{\circ} \mathrm{C}$ in air, following the solid state reaction routine way. Slight excess of $\mathrm{Bi}_{2} \mathrm{O}_{3}$ has been added to compensate the preferential loss of bismuth during sintering. The XRD result of the target material indicates that the sample is in single phase of polycrystalline BFMO.

Before deposition, the $\mathrm{Pt}(111) / \mathrm{Ti} / \mathrm{SiO}_{2} / \mathrm{Si}(100)$ substrate was preheated at $700^{\circ} \mathrm{C}$ to eliminate surface contamination. The substrate temperature and oxygen ambient pressure were maintained at $550^{\circ} \mathrm{C}$ and $5.6 \mathrm{~Pa}$, respectively. During the deposition, the laser fluence was at $2.3 \mathrm{~J} / \mathrm{cm}^{2}$ and the pulse repetition rates $1.5 \mathrm{~Hz}$. After deposition, all the films were annealed at same condition for 30 minutes and then transferred under a background pressure of $\sim 10^{-8} \mathrm{~Pa}$ to the PES chamber to carry out in situ XAS measurements. The overall energy resolution was $0.2-0.7 \mathrm{eV}$, depending on the photon energies. All the spectra were recorded at room temperature, and the XAS measurement was carried out with total electron yield (TEY) detection mode. After the in situ measurements, the films were taken for ex situ crystal structure characterization by XRD, surface morphology by SEM, and magnetic AGM measurements.

\section{Result and Discussion}

3.1. Structure and Microstructure Characterization. XRD spectra were recorded at room temperature on an (MSAL$\mathrm{XD} 2) \mathrm{X}$-ray diffractometer with a $\mathrm{Cu} \mathrm{K}_{\alpha}$ source $(\alpha=$ $1.5418 \AA$ ) in Bragg-Brentano geometry. Figure 1(a) shows the X-ray diffraction patterns of $\mathrm{BiFe}_{1-x} \mathrm{Mn}_{x} \mathrm{O}_{3}(x=0,0.1$, 0.2 , and 0.3$)$ thin films deposited on $\mathrm{Pt}(111) / \mathrm{Ti} / \mathrm{SiO}_{2} / \mathrm{Si}(100)$ substrate without buffer layers. The BFO films, which are directly deposited on the Pt-buffered substrates, often exhibit a polycrystalline structure with one or more secondary phases $[12,13]$. In comparison, in this work all the films are in single-phase polycrystalline structure with rhombohedral space group $(R 3 \mathrm{c})$ and no other peak related to secondary phases was detected. It is also interesting to notice that a varied Mn substitution results in the regulation of the crystal orientation of the $\mathrm{BiFe}_{1-x} \mathrm{Mn}_{x} \mathrm{O}_{3}$ thin films. Figure $1(\mathrm{~b})$ shows the enlarged XRD patterns of films in the 2-theta range $20 \sim 24$. Relative to that of pure BFO film, the (100) peaks of Mn doped show a slight shift to higher angle with a similar peak shapes and structures, confirming that the $\mathrm{Fe}$ ion substitution by Mn in BFO films. Except the rigid shift of the peak positions of doped sample compare to the undoped one, and a slight modulation of the (100) peak width in the doping range, crystalline structure of BFO has been affected little by the substitutional doping of $\mathrm{Mn}$, in agreement with the rhombohedral structure of BFO unchanged up to $30 \%$ of Mn doping $[5,10]$.

The morphological SEM images of the $\mathrm{BiFe}_{1-x} \mathrm{Mn}_{x} \mathrm{O}_{3}$ $(x=0,0.1,0.2,0.3)$ thin films on $\mathrm{Pt}(111) / \mathrm{Ti} / \mathrm{SiO}_{2} / \mathrm{Si}(100)$ substrate are shown in Figure 2. The SEM image of undoped BFO film, in Figure 2(a), reveals nonuniform growth of grains with wide pores between grains and an averagely different grain sizes. The Mn doping significantly affects the morphologies of BFMO thin films (Figure 2(b), 2(c), and $2(\mathrm{~d}))$, the films become denser, the grain sizes tend to be smaller in (b) and (c), the grain boundaries are blurred, and the pores between grains are reduced. Larger pores between the grains of undoped and 30\% Mn-doped thin films are consistent with the weaker diffraction intensity of those films in Figure 1(a).

The XRD peaks and the SEM images, as direct indicators for atomic long-range ordering level and macroscopic morphology of crystal, both provide mutual-revealing clues on what has happened in atomistic level of the systems along with variational condition, such as doping in the underlying case. The grain size variation observed in the SEM images shows a consistent relation with the peak width in the XRD; that is, the larger the grain sizes are, the wider the observed XRD peaks are, though the latter at a poorer statistical level. When all the samples keeping the phase of crystal structure within the same R3c symmetry group, the facts such as the rigid peak positions shift of (100), gradual disappearance of peak (110), and the various morphological show-up of the crystal films against the Mn doping imply the systems undergone certain kind of electronic structural modulation, instead of the results from simple mixing of different radius ball-like elements.

Change in $2 \theta$ implies a change in d-spacing of lattice parameters. The (100) lattice parameter $a$ and the diffraction $2 \theta$ angle have the relation $\propto 1 / \sin \theta$ the small amount upshift of the $2 \theta$ implies a decrease in the $a$ with the Mn doping. The rigidness of the shift by reaching a fixed position all at once reflects that, first of all, the sensitivity of the electronic structure to the doping rather than the doping amount, secondly, the dopant distribution uniformity of the substitution. We attribute the d-spacing decrease and the rigid shift of $2 \theta$ to electronic structural effects, rather than a different size elemental mixing effects. If it was the latter effects, as it has been shown clearly for Fe-Al mixing in [14], the smaller size Fe substitution of larger size Al drove a continuous decrease of lattice parameters with their relative percentage, in the underlying case we would have observed a scenario where the lattice parameters were increasing and the $2 \theta$ degree decreasing continuously following the fact that 


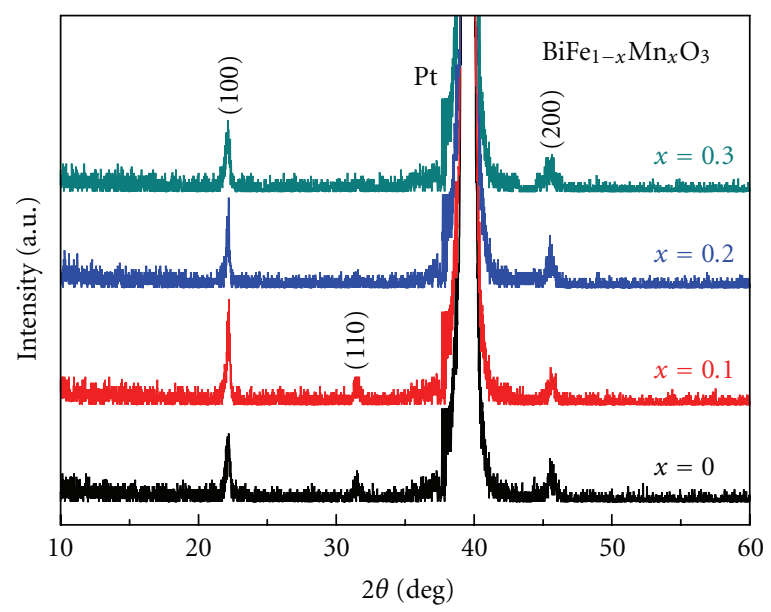

(a)

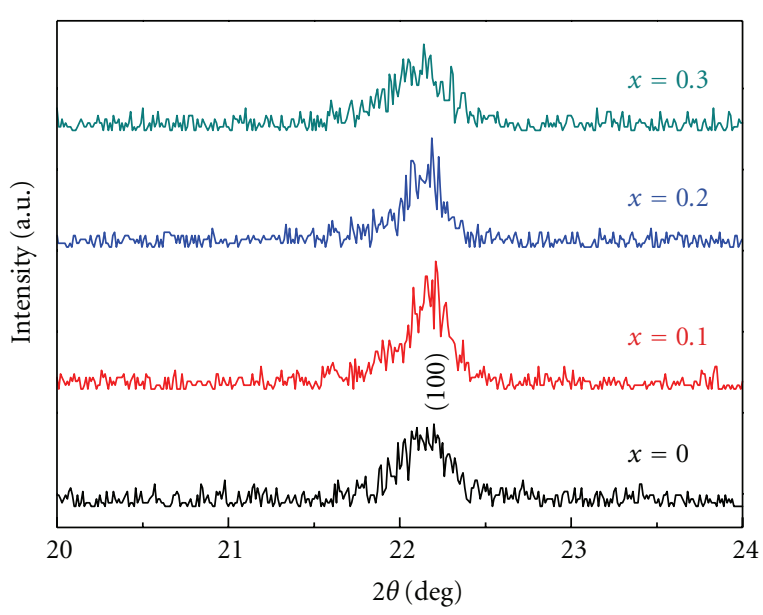

(b)

Figure 1: X-ray diffraction (XRD) patterns of $\mathrm{BiFe}_{1-x} \mathrm{Mn}_{x} \mathrm{O}_{3}(x=0,0.1,0.2,0.3)$ films on $\mathrm{Pt}(111) / \mathrm{Ti} / \mathrm{SiO}{ }_{2} / \mathrm{Si}(100)$ substrate: (a) $2 \theta$ from 10 to 60 ; (b) enlarged $2 \theta$ of (100) from 20 to 24.

the $\mathrm{Mn}$ atom has a larger radius than that of Fe. All these are clearly in a contrary way of the above simple elemental situation. The disappearance of the (110) indexing surface with the Mn doping can be taken to be a direct result in the electronic structural regulation. This can be due to randomization, distortion or breakdown of the (110) surface, but certainly not due to, continuously changing grain sizes; this is not the case shown in the measured SEM images. The electronic structural effects in the underlying case are mostly by modulation of the hybridization interactions between $\mathrm{Fe}-\mathrm{O}$ and Mn-O two pair elements, as they are discussed through the XAS results at $\mathrm{Fe} L$ edge and $\mathrm{O} K$ edge in the following sections. The observed electronic structural variations with doping provide an affirmative standing point for the XRD and SEM observations.

3.2. Fe L Edge X-Ray Absorption Spectroscopy. The Fe $2 p$ XAS spectra of BFMO system in Figure 3(a), along with the spectra of reference trivalent $\mathrm{Fe}_{2} \mathrm{O}_{3}\left(\mathrm{Fe}^{3+}\right)$ and divalent $\mathrm{FeO}$ $\left(\mathrm{Fe}^{2+}\right)$ oxides [15], provide information on the unoccupied Fe $3 d$ states. The Fe $3 d$ orbitals spilt by exchange interaction into majority (spin up $\uparrow$ ) and minority (spin down $\downarrow$ ) states. Each state further splits by the octahedral crystal ligand field into $t_{2 g}$ and $e_{g}$ orbitals. In the Fe $L$ edges XAS spectra, the lower energy shoulder of Fe $L_{3}$ corresponds to the unoccupied $t_{2 g} \downarrow$ states and the sharper one to the unoccupied $e_{g} \downarrow$ states. Similar to $L_{3}$ edge, the $L_{2}$ edge also splits into two $t_{2 g} \downarrow$ and $e_{g} \downarrow$ states. The energy separation between $t_{2 g} \downarrow$ and $e_{g} \downarrow$ states is called octahedral crystal field $10 \mathrm{Dq}$ value (or the distance between the $t_{2 g}$ and $e_{g}$ ). Measured energy separation between $t_{2 g} \downarrow$ and $e_{g} \downarrow$ states for all the samples is $\sim 1.6 \mathrm{eV}$. This leads the Fe $3 d$ electrons to stay at high-spin configuration $t_{2 g}{ }^{3} e_{g}{ }^{2}\left({ }^{6} \mathrm{~A}_{1 g}\right)$, like the Fe $3 d$ electrons in $\mathrm{LaFeO}_{3}$.

The line shape of the $\mathrm{Fe} 2 p$ XAS provides information on the valence state of the Fe ions. The spectra show that the line shapes of all the doped samples are similar to that of $\mathrm{Fe}_{2} \mathrm{O}_{3}$ and quite different from that of $\mathrm{FeO}$. These indicate that the Fe ions keep formally in trivalent $\left(\mathrm{Fe}^{3+}\right)$ states with the doping, consistent with literature report [16].

The change in peak intensity and peak width reflects a modulation in the electron density of state distribution between the $\mathrm{Fe} 3 d$ and ligand $\mathrm{O} 2 p$ with doping. In Figure 3(b), the $t_{2 g} \downarrow$ peak weight estimated by the $I_{t_{2 g}} /\left(I_{t_{2 g}}+\right.$ $I_{e_{g}}$ ) intensity ratio and the $L_{3}$ edge FWHM (full width at half maximum) by arithmetic sum of $t_{2 g} \downarrow$ and of $e_{g} \downarrow$ peaks' FWHM show explicit variation trends. As $x$ increases, the intensity of $t_{2 g} \downarrow$ state increases from 0.364 at $x=0$ to 0.408 at $x=0.3$; at the same time, the $L_{3}$ edge peak width shrinks from $1.63 \mathrm{eV}$ to $1.41 \mathrm{eV}$. Origin for these variations can be understood in terms of the doping-driven hybridization strength modification between the $\mathrm{Fe} 3 d$ and $\mathrm{O} 2 p$ states. The starting point is put on the valence band state of the $x=0$ undoped BFO system. The BFO system is assumed to be $\mathrm{Fe} 3 d^{5}+\mathrm{Fe} 3 d^{6} \underline{L}$ ( $\underline{L}$ represents the ligand hole state O $2 p^{5} \underline{L}$ formed by a charge transfer from $\mathrm{O} 2 p^{6}$ to $\mathrm{Fe} 3 d^{5}$ ) mixture states derived by the Fe $3 d$ and $\mathrm{O} 2 p$ hybridization. The Fe $3 d^{6} \underline{L}$ state is induced by charge transfer from the ligand $\mathrm{O} 2 p$ to $\mathrm{Fe} 3 d$. The $t_{2 g} \downarrow$ state intensity change indicates an increase of the $\mathrm{Fe} 3 d^{5}$ portion with the doping and correspondingly implies a decrease of the Fe $3 d^{6} \underline{L}$ charge transfer state. The simultaneous peak width narrowing can be discussed analogously to the intensity change as result of the hybridization strength decrease. The $\mathrm{O} 2 p$ ligand valence band is of 5.5 to $6 \mathrm{eV}$ wide [17]; a stronger hybridization effect in the pure BFO induces higher portion of Fe $3 d^{6} \underline{L}$ in the $\mathrm{Fe} 3 d^{5}+\mathrm{Fe} 3 d^{6} \underline{L}$ mixed state then gives the observed weaker intensity and larger peak width due to delocalization. At the same time, the reduced hybridization effect along the Mn doping brings about an increase of the Fe $3 d^{5}$ portion in the $\mathrm{Fe} 3 d^{5}+\mathrm{Fe} 3 d^{6} \underline{L}$ states. Main causes of the current observation at the Fe $L$ edge, in a self-consistent way with an increased hybridization strength between $\mathrm{Mn} 3 d-\mathrm{O} 2 p$, are clearly seen affirmatively through the below $\mathrm{O} K$ edge XAS measurement results. 


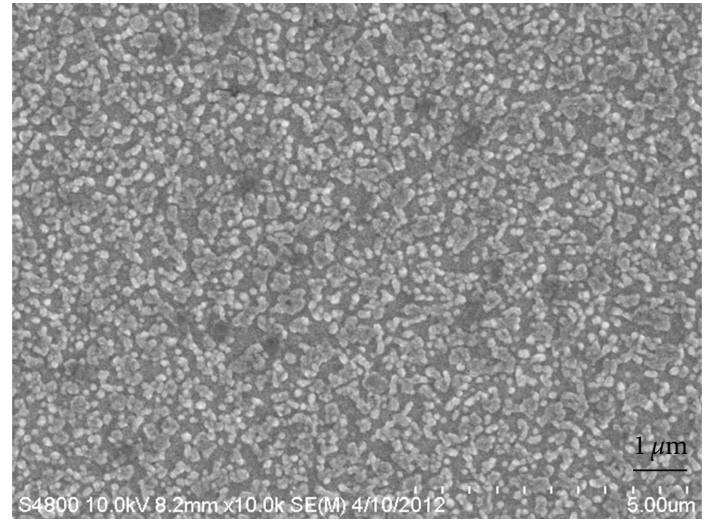

(a)

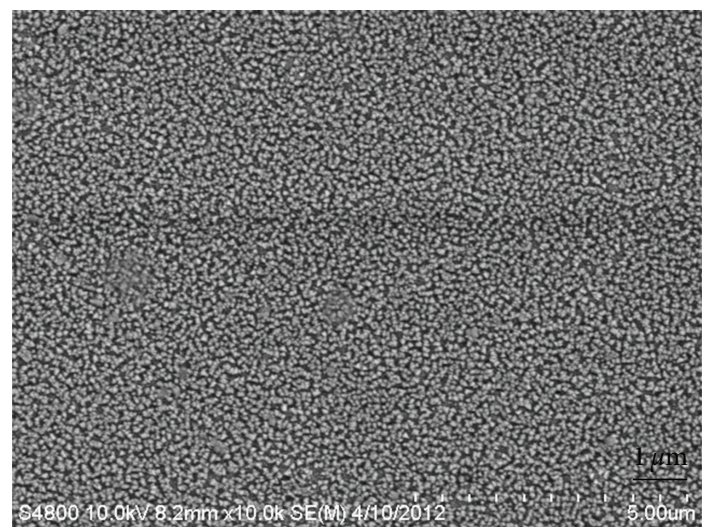

(c)

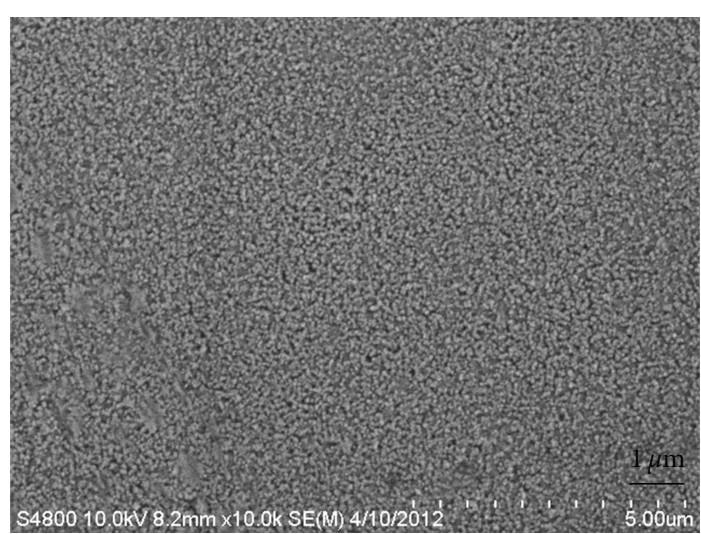

(b)

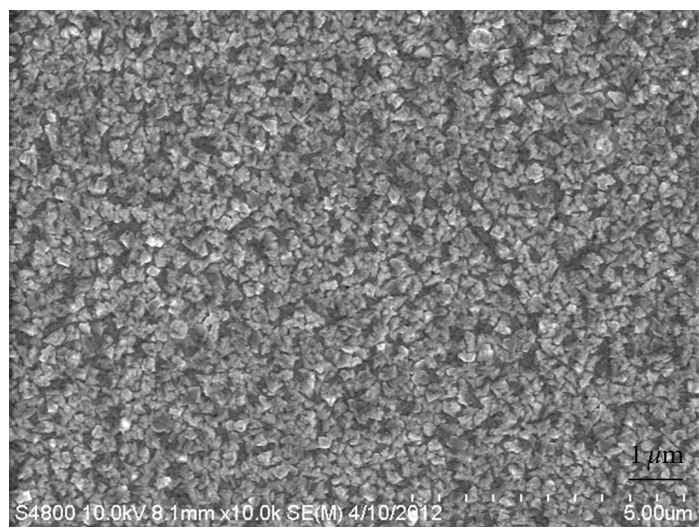

(d)

FIGURE 2: SEM images of $\mathrm{BiFe}_{1-x} \mathrm{Mn}_{x} \mathrm{O}_{3}(x=0,0.1,0.2,0.3)$ films with the composition of (a) $x=0$; (b) $x=0.1 ;$ (c) $x=0.2$; (d) $x=0.3$ prepared on $\mathrm{Pt}(111) / \mathrm{Ti} / \mathrm{SiO}_{2} / \mathrm{Si}(100)$ substrate. The images are obtained with weak contrast due to fast saturation charging effect.

3.3. Oxygen K Edge X-ray Absorption Spectroscopy. The O Kedge XAS spectra, shown in Figure 4(a), are labeled a, b, and c. The relative intensity change of the peak a's shoulder and the FWHM of the double-peak a, as shown in Figure 4(b), are obtained with the same way as that of the Fe $2 p$ XAS results shown in Figure 3(b). The $\mathrm{M} 3 d^{n+1} \underline{L}$ characteristic peak intensity increases from $\sim 0.1$ to $\sim 0.21$ with the $\mathrm{Mn}$ content change 0 through 0.3 , and at the same time the peaks' width decreases from $1.95 \mathrm{eV}$ to $1.25 \mathrm{eV}$.

The O $1 s$ XAS measurement provides holistic information on the $\mathrm{O} 2 p$ hybridization with transition metals $\mathrm{M} 3 d^{n}$ $+\mathrm{M} 3 d^{n+1} \underline{L}$ states where the M $3 d^{n}+\mathrm{M} 3 d^{n+1} \underline{L}$ represents the $\left[\mathrm{Fe} 3 d^{5}+\mathrm{Fe} 3 d^{6} \underline{L}\right]+\left[\mathrm{Mn} 3 d^{4}+\mathrm{Mn} 3 d^{5} \underline{L}\right]$ mixture states. The spectral structure reflects the density of states of $\mathrm{M}$ $3 d^{n+1} \underline{L}$ portion in $\mathrm{M} 3 d^{n}+\mathrm{M} 3 d^{n+1} \underline{L}$ mixed states, since the hole state $\underline{L}$ of $\mathrm{O} 2 p$ characteristic orbitals results in the charge transfer via hybridization interactions between $\mathrm{M} 3 d$ and $\mathrm{O} 2 p$. The a double-peak structure including the arrow pointing shoulder is of the $\mathrm{O} 2 p$ characteristic $\mathrm{M} 3 d^{n+1} \underline{L}$ part in the $\mathrm{M} 3 d^{n}+\mathrm{M} 3 d^{n+1} \underline{L}$ states and these states are about the shoulder $t_{2 g} \downarrow$ and the main peak $e_{g} \downarrow$ states, respectively [18]. When the density of the hole-state $\underline{L}$ at ligand side is high, the higher the intensity of peaks' $a$ is observable. The band $\mathrm{b}$ is attributed to the hybridization of $\mathrm{O} 2 p$ with $\mathrm{Bi} 6 s p$ states and the $\mathrm{c}$ at $\sim 542 \mathrm{eV}$ to the hybridization of $\mathrm{O} 2 p$ with $\mathrm{Fe} 4 s p$, Mn $4 s p$ orbitals.

In this section we pay special attention to the doublepeak a due to it is about the $\mathrm{M} 3 d^{n+1} L$ state. The above $\mathrm{Fe}$ $2 p$ XAS results, which are for the Fe $3 d^{5}$ portion of Fe $3 d^{5}$ $+\mathrm{Fe} 3 d^{6} \underline{L}$ mixed states, have reached the conclusion that the $\mathrm{Fe} 3 d^{6} \underline{L}$ density of state decreases and correspondingly the $\mathrm{Fe}$ $3 d^{5}$ state increases with the $\mathrm{Mn}$ doping. Now at $\mathrm{O} K$ edge, we see that the $\mathrm{M} 3 d^{n+1} \underline{L}$ characteristic peak intensity increases, and simultaneously the peaks' width decreases with the $\mathrm{Mn}$ doping, as shown in Figure 4(b).

At the $\mathrm{O} K$ edge, the increase of $t_{2 g} \downarrow$ characteristic M $3 d^{n+1} \underline{L}$ intensity with Mn doping reflects the fact that available $\underline{L}$ states at the ligand side are increasing, and this is by an enhanced hybridization effect which drives the charge transfer from ligand to metal ions. The $\mathrm{M}$ $3 d^{n+1} \underline{L}$ state includes contributions from both states $\mathrm{Fe}$ $3 d^{6} \underline{L}$ and $\mathrm{Mn} 3 d^{5} \underline{L}$. In the underlying system, the decrease of Fe $3 d^{6} \underline{L}$ density of state and correspondingly the increase of Fe $3 d^{5}$ with Mn have been proved by the XAS results at Fe $L$ edge. The intensity increase of the $\mathrm{M} 3 d^{n+1} \underline{L}$ state at $\mathrm{O} K$ edge, then, uniquely corresponds to the increase of Mn $3 d^{5} \underline{L}$ density of state, and simultaneously a decrease of Mn $3 d^{4}$ state with the doping. The double-peak width 


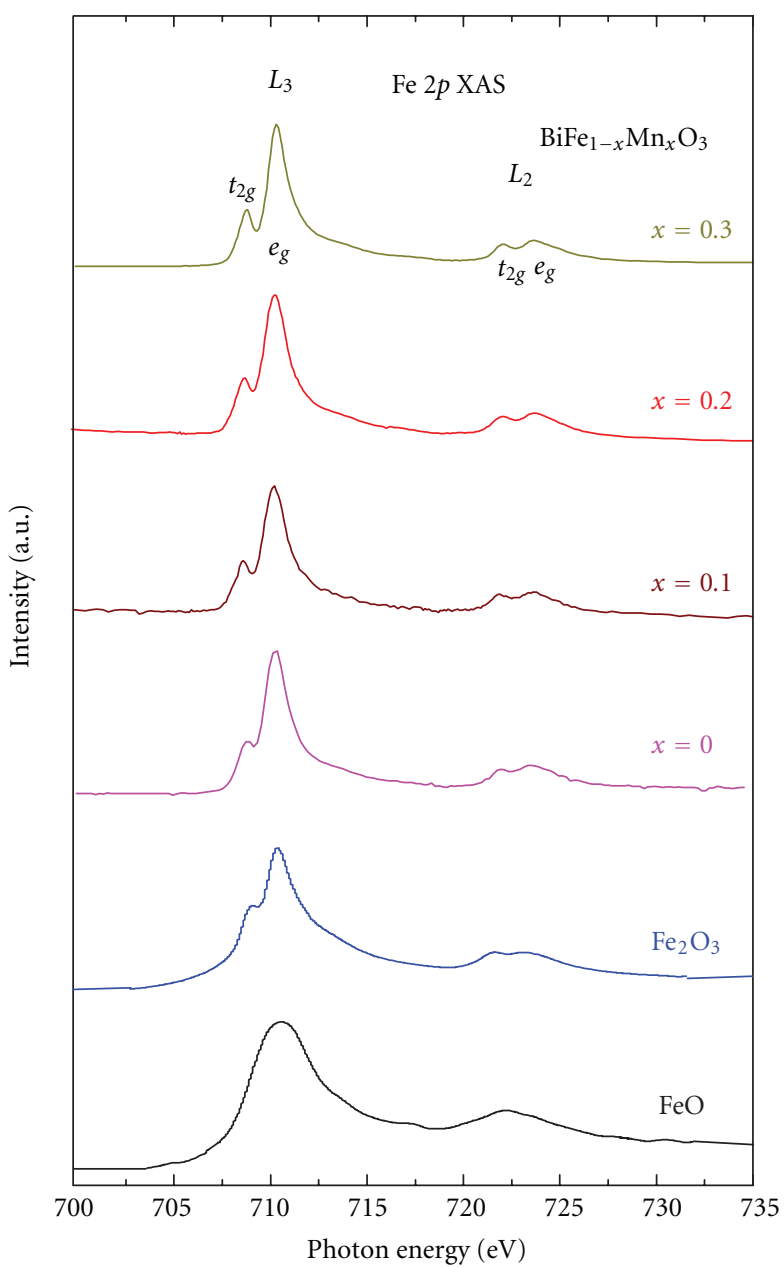

(a)

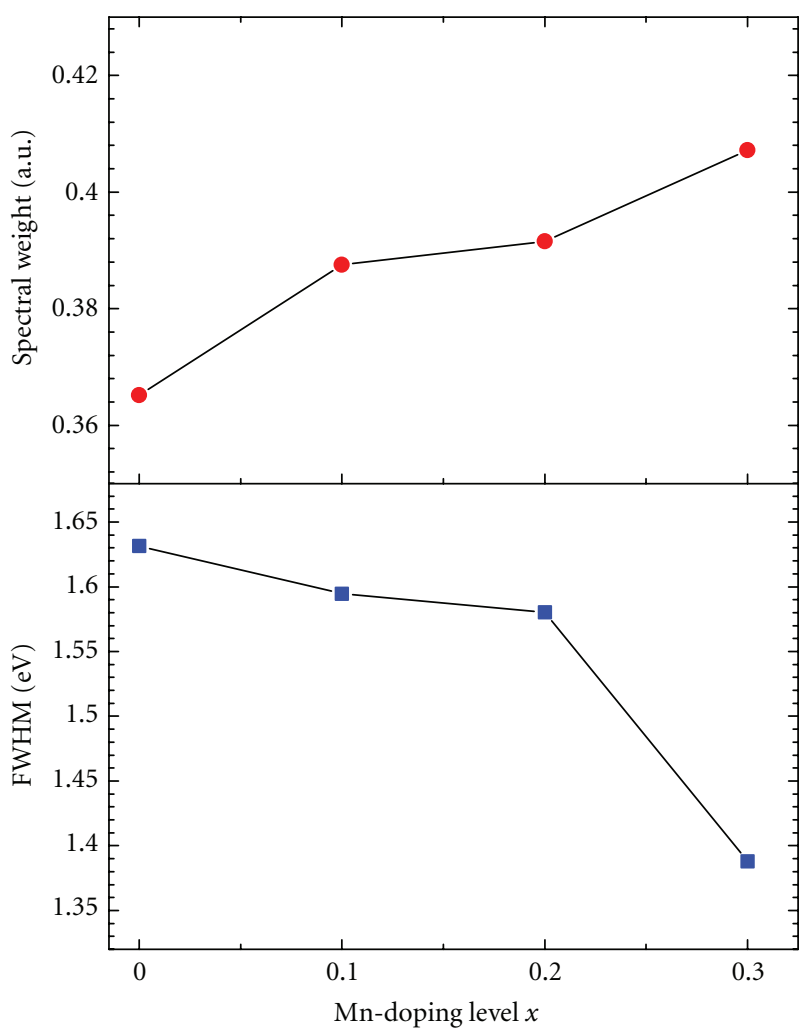

- Spectral weight of $t_{2 g}$ in $L_{3}$ edge

- FWHM of $L_{3}$ edge

Figure 3: (a) Comparison of the Fe $2 p$ XAS of $\mathrm{BiFe}_{1-x} \mathrm{Mn}_{x} \mathrm{O}_{3}(0 \leq x \leq 0.3)$ with those of $\mathrm{FeO}$ and $\mathrm{Fe}_{2} \mathrm{O}_{3}$; (b) FWHM of $L_{3}$ edge and spectral weight of $\mathrm{t}_{2 g}$ in $L_{3}$ edge as a function of $x$.

decrease at the $\mathrm{O} K$ edge implies that the localization of $\mathrm{Mn}$ $3 d^{5} \underline{L}$ states is enhanced with the Mn doping. These results indicate a scenario that the $\mathrm{Fe} 3 d-\mathrm{O} 2 p$ hybridization strength weakens with the enhanced $\mathrm{Mn} 3 d-\mathrm{O} 2 p$ hybridization effect in the BFMO thin films.

3.4. Magnetic Properties. The magnetic hysteresis (M-H) curves of the films measured at room temperature are shown in Figure 5. The $\mathrm{M}-\mathrm{H}$ curves indicate typical ferromagnetic characters. The pure BFO thin film shows a weak ferromagnetic behavior in slim magnetic loops (Ms) of $28.7 \mathrm{emu} / \mathrm{cm}^{3}$, since it is in a rhombohedral distorted perovskite structure and allows a weak ferromagnetic ordering due to canting of the spins [19]. The magnetization value of pure BFO films in this work almost same with other reports [7]. The Ms of the samples at $x=0.1, x=0.2$, and $x=0.3$ are $31.2,40.3$, and $47.7 \mathrm{emu} / \mathrm{cm}^{3}$, respectively (inset of Figure 5). These values are higher than those of their corresponding bulk samples [20]. This increasing trend of Ms through Mn doping agrees with that of other reports about BFMO thin films and ceramics $[11,21,22]$. The coercive field $\left(\mathrm{H}_{C}\right)$ decreases with the increase of Mn composition.

The increase in macroscopic magnetization with the increase of Mn content (inset of Figure 5) was attributed to either structural transition or exhibition of certain amount of $\mathrm{Fe}^{2+} / \mathrm{Fe}^{3+}$ mixed ions in films $[9,11]$. XPS results (not shown here) and the Fe $L$ edge XAS show formal $\mathrm{Fe}^{3+}$ only composition and no trace of $\mathrm{Fe}^{2+}$ demonstrating. From the XRD results, however, there is no clear evidence for a phase transition such as from rhombohedral to tetragonal or other phases with Mn doping. These imply the BFMO thin films magnetization enhancement could not be from the different valence states of $\mathrm{Fe}$ ions. It also does not originate from the misfit strain by different films thickness $[7,23]$, since all the BFMO films were grown with a same film thickness, that is, the films deposition rates monitored in situ by crystal oscillator during growth process. Enhancement of the Ms with Mn doping is actually due to change in the electron density of state distributions between $\mathrm{Fe}-\mathrm{O}$ and $\mathrm{Mn}-\mathrm{O}$ pairs through variation of hybridization interactions, as discussed in section below. 


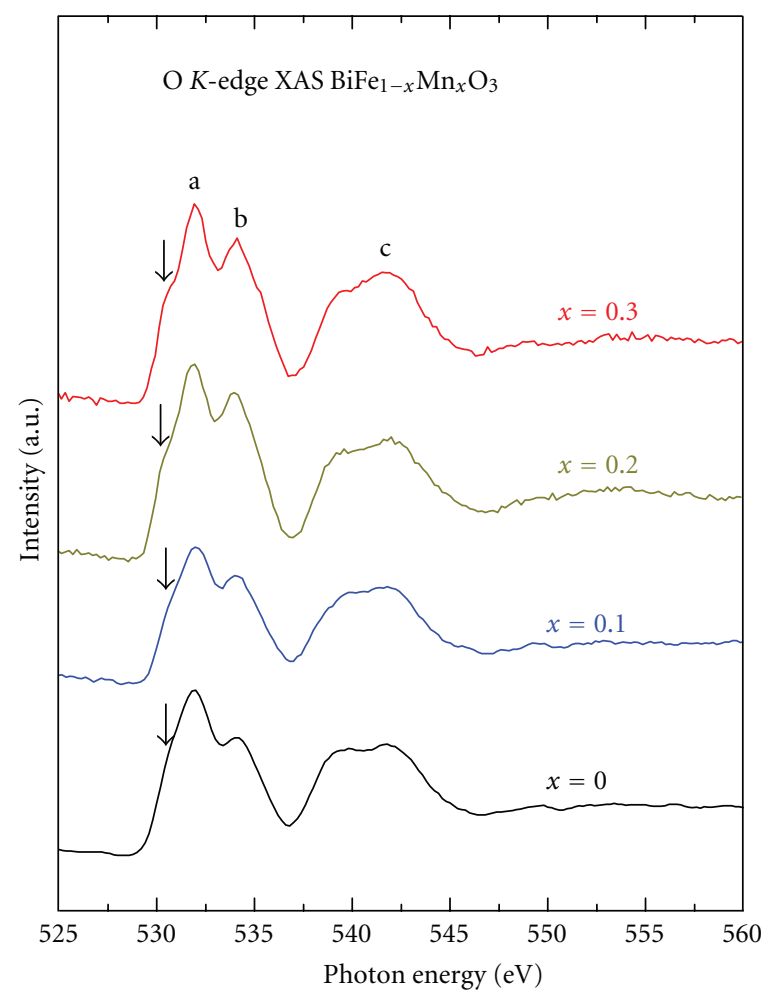

(a)

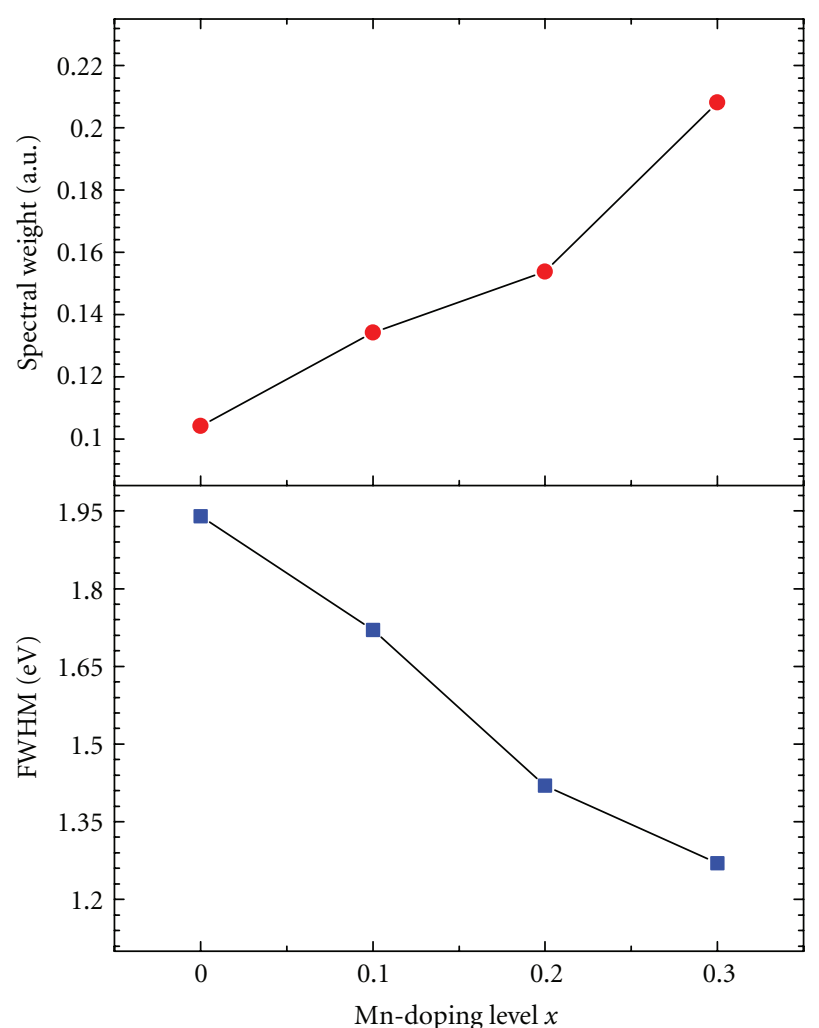

Spectral weight of shoulder in peak a

FWHM of peak a

(b)

Figure 4: (a) $\mathrm{O} K$-edge XAS spectra of $\mathrm{BiFe}_{1-x} \mathrm{Mn}_{x} \mathrm{O}_{3}$ thin films with $x=0,0.1,0.2$, and 0.3 doping levels. (b) Spectral weight of the shoulder in peak a and the FWHM of peak a as function of $x$.

Concerning the magnetic property of doped ceramic system, in an early work on the role of covalency in magnetic property of an A-site-doped $\mathrm{ABO}_{3}$ (e.g., $\mathrm{La}^{3+}$ substitution with $\mathrm{Sr}^{2+}$ in the $\mathrm{LaMnO}_{3}$ ) perovskite-type manganite $\mathrm{La}_{1-x} \mathrm{Sr}_{x} \mathrm{MnO}_{3}$ [24], Goodenough assumed that inhomogeneity in the bonding nature of the central cations $\mathrm{Mn}^{3+}$ and $\mathrm{Mn}^{4+}$ with anion $\mathrm{O}^{2-}$ by forming a mixed state of ionic, covalent, or semicovalent bonds could be the major driving force for ferromagnetic property observed in the system. The basic idea behind is that if the $\mathrm{Mn}^{3+}-\mathrm{O}^{2-}$ bond has more ionic character and the $\mathrm{Mn}^{4+}-\mathrm{O}^{2-}$ with more covalency or semicovalency, the bonding inhomogeneity with greater separation for ionic bonding and shorter for covalence will ease the barrier of ferromagnetic moment in the $\mathrm{Mn}^{3+}\left(3 \mathrm{~d}^{4}\right)-\mathrm{O}^{2-}-\mathrm{Mn}^{4+}\left(3 \mathrm{~d}^{3}\right)$ chain. The observed ferromagnetic results in the underlying BFMO system in the $\mathrm{Mn}^{3+}\left(3 \mathrm{~d}^{4}\right)-\mathrm{O}^{2-}-\mathrm{Fe}^{3+}\left(3 \mathrm{~d}^{5}\right)$ chain, analogous to the case suggested for the $\mathrm{Mn}^{3+}\left(3 \mathrm{~d}^{4}\right)-\mathrm{O}^{2-}-\mathrm{Mn}^{4+}\left(3 \mathrm{~d}^{3}\right)$ chain, demonstrate a good agreement to the assumption, showing an increase of ionic bond character for $\mathrm{Fe}^{3+}-\mathrm{O}^{2-}$ as observed at $\mathrm{Fe} 2 p$ edge XAS and an increase of covalence bonding for $\mathrm{Mn}^{3+}-\mathrm{O}^{2-}$ at $\mathrm{O} K$ edge XAS. Apparently, the increase of the magnetism in the underlying system with the Mn doping associates closely to the bonding inhomogeneity created in the $\mathrm{Mn}^{3+}\left(3 \mathrm{~d}^{4}\right)-\mathrm{O}^{2-}-\mathrm{Fe}^{3+}\left(3 \mathrm{~d}^{5}\right)$ chains with the Mn doping.

\section{Conclusion}

In summary, the polycrystalline multiferroic $\mathrm{BiFe}_{1-x} \mathrm{Mn}_{x} \mathrm{O}_{3}$ $(0 \leq x \leq 0.3)$ thin films were grown by PLD techniques. The X-ray diffraction spectroscopy shows Mn content up to $x=0.3$ is in single phase having a rhombohedral crystalline structure. The crystal structural variation with Mn doping is attributed to the electronic structural modulation, instead of different size elemental mixing. Mn doping brings about an increase of the $\mathrm{Fe} 3 d^{5}$ portion in the $\mathrm{Fe} 3 d^{5}+\mathrm{Fe}$ $3 d^{6} \underline{L}$ states and spontaneous decrease of $\mathrm{Mn} 3 d^{4}$ portion in the $\mathrm{Mn} 3 d^{4}+\mathrm{Mn} d^{5} \underline{L}$ mixed states. The observed increase in Ms with increasing Mn concentration was closely related with the electronic density of state distribution changes. Increase in the $\mathrm{Mn}$ concentration reduces the charge transfer between the $\mathrm{Fe} 3 d$ and $\mathrm{O} 2 p$ and leads to the bond inhomogeneity in the $\mathrm{Fe}-\mathrm{O}-\mathrm{Mn}$ chains; these change driven by the hybridization interactions are responsible for the magnetization enhancement, as well as the observed slight modulation in crystal structures. 


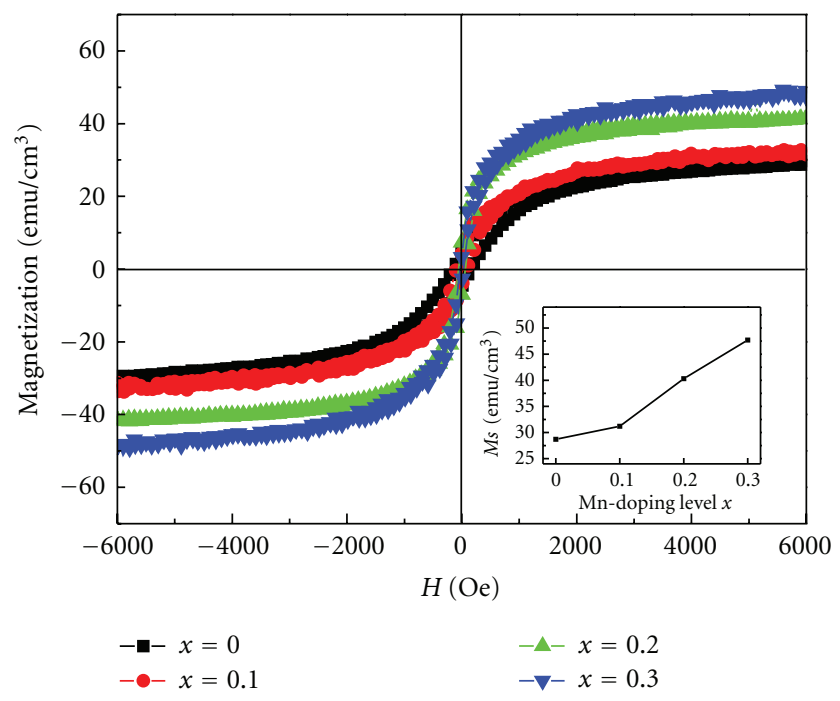

Figure 5: Magnetic hysteresis (M-H) curves measured at room temperature for the $\mathrm{BiFe}_{1-x} \mathrm{Mn}_{x} \mathrm{O}_{3}$ thin films with $x=0,0.1$, 0.2 , and 0.3 . The inset shows the magnetization (Ms) plotted as a function of Mn-doping level.

\section{Acknowledgments}

The authors acknowledge the financial support from Chinese Academy of Sciences under Grant no. H91G750Y21, and National Natural Science Foundation of China (Grant no. 11164026).

\section{References}

[1] W. Eerenstein, N. D. Mathur, and J. F. Scott, "Multiferroic and magnetoelectric materials," Nature, vol. 442, no. 7104, pp. 759-765, 2006.

[2] M. Fiebig, T. Lottermoser, D. Frohlich, A. V. Goltsev, and R. V. Pisarev, "Observation of coupled magnetic and electric domains," Nature, vol. 419, no. 6909, pp. 818-820, 2002.

[3] N. A. Hill, "Why are there so few magnetic ferroelectrics?" Journal of Physical Chemistry B, vol. 104, no. 29, pp. 66946709, 2000.

[4] R. Ramesh and N. A. Spaldin, "Multiferroics: progress and prospects in thin films," Nature Materials, vol. 6, no. 1, pp. 2129, 2007.

[5] C. H. Yang, T. Y. Koo, and Y. H. Jeong, "How to obtain magnetocapacitance effects at room temperature: the case of $\mathrm{Mn}$-doped $\mathrm{BiFeO}_{3}$," Solid State Communications, vol. 134, no. 4, pp. 299-301, 2005.

[6] M. S. Kartavtseva, O. Y. Gorbenko, A. R. Kaul, and T. V. Murzina, "The influence of epitaxial strain on magnetic and electrical properties of $\mathrm{BiFeO}_{3}$ thin films," Thin Solid Films, vol. 518, no. 16, pp. 4750-4752, 2010.

[7] F. Z. Huang, X. M. Lu, W. W. Lin et al., “Thickness-dependent structural and magnetic properties of $\mathrm{BiFeO}_{3}$ films prepared by metal organic decomposition method," Applied Physics Letters, vol. 97, no. 22, Article ID 222901, 2010.

[8] A. M. dos Santos, S. Parashar, A. R. Raju, Y. S. Zhao, A. K. Cheetham, and C. N. R. Rao, "Evidence for the likely occurrence of magnetoferroelectricity in the simple perovskite, $\mathrm{BiMnO}_{3}$," Solid State Communications, vol. 122, no. 1-2, pp. 49-52, 2002.

[9] K. Takahashi and M. Tonouchi, "Influence of Mn doping on ferroelectric-antiferromagnet $\mathrm{BiFeO}_{3}$ thin films grown on $\left(\mathrm{LaAlO}_{3}\right)_{0.3}\left(\mathrm{Sr}_{2} \mathrm{AlTaO}_{6}\right)_{0.7}$ substrates," Japanese Journal of Applied Physics Part 2, vol. 45, no. 29-32, pp. L755-L757, 2006.

[10] M. Kumar and K. L. Yadav, "Rapid liquid phase sintered Mn doped $\mathrm{BiFeO}_{3}$ ceramics with enhanced polarization and weak magnetization," Applied Physics Letters, vol. 91, no. 24, Article ID 242901, 2007.

[11] J. J. Gu, G. L. Zhao, F. W. Cheng, J. R. Han, L. H. Liu, and H. Y. Sun, "Magnetoelectric properties of $\mathrm{Mn}$-substituted $\mathrm{BiFeO}_{3}$ thin films with a $\mathrm{TiO}_{2}$ barrier," Physica B, vol. 406, no. 23, pp. 4400-4403, 2011.

[12] M. H. Lee, J. S. Park, D. J. Kim et al., "Ferroelectric properties of $\mathrm{Mn}$-doped $\mathrm{BiFeO}_{3}$ thin films," Current Applied Physics, vol. 11, no. 3, supplement, pp. S189-S192, 2011.

[13] T. Kawae, Y. Terauchi, H. Tsuda, M. Kumeda, and A. Morimoto, "Improved leakage and ferroelectric properties of $\mathrm{Mn}$ and Ti codoped $\mathrm{BiFeO}_{3}$ thin films," Applied Physics Letters, vol. 94, no. 11, Article ID 112904, 2009.

[14] B. Huang, K. N. Ishihara, and P. H. Shingu, "Metastable phases of Al-Fe system by mechanical alloying," Materials Science and Engineering A, vol. 231, no. 1-2, pp. 72-79, 1997.

[15] J. P. Crocombette, M. Pollak, F. Jollet, N. Thromat, and M. Gautier-Soyer, "X-ray-absorption spectroscopy at the Fe $L_{2,3}$ threshold in iron oxides," Physical Review B, vol. 52, no. 5, pp. 3143-3150, 1995.

[16] G. Anjum, R. Kumar, S. Mollah, P. Thakur, S. Gautam, and K. H. Chae, "NEXAFS studies of $\mathrm{La}_{0.8} \mathrm{Bi}_{0.2} \mathrm{Fe}_{1-x} \mathrm{Mn}_{x} \mathrm{O}_{3}(0.0 \leq x \leq$ $0.4)$ multiferroic system using $\mathrm{X}$-ray absorption spectroscopy," Journal of Physics D, vol. 44, no. 7, Article ID 075403, 2011.

[17] R. L. Kurtz and V. E. Henrich, "Surface electronic structure and chemisorption on corundum transition-metal oxides: $\mathrm{V}_{2} \mathrm{O}_{3}$," Physical Review B, vol. 28, no. 12, pp. 6699-6706, 1983.

[18] R. Sæterli, S. M. Selbach, P. Ravindran, T. Grande, and R. Holmestad, "Electronic structure of multiferroic $\mathrm{BiFeO}_{3}$ and related compounds: electron energy loss spectroscopy and density functional study," Physical Review B, vol. 82, no. 6, Article ID 064102, 2010.

[19] R. Ramesh, J. Wang, J. B. Neaton et al., "Epitaxial $\mathrm{BiFeO}_{3}$ multiferroic thin film heterostructures," Science, vol. 299, no. 5613, pp. 1719-1722, 2003.

[20] Q. Y. Xu, S. Q. Zhou, Z. Wen et al., "Magnetic characterization of $\mathrm{Bi}\left(\mathrm{Fe}_{1-x} \mathrm{Mn}_{x}\right) \mathrm{O}_{3}$," Physics Letters A, vol. 375, no. 8, pp. 1209-1212, 2011.

[21] L. H. Yin, Y. P. Sun, F. H. Zhang et al., "Magnetic and electrical properties of $\mathrm{Bi}_{0.8} \mathrm{Ca}_{0.2} \mathrm{Fe}_{1-x} \mathrm{Mn}_{x} \mathrm{O}_{3}(0 \leq x \leq 0.5)$," Journal of Alloys and Compounds, vol. 488, no. 1, pp. 254-259, 2009.

[22] M. Azuma, H. Kanda, A. A. Belik, Y. Shimakawa, and M. Takano, "Magnetic and structural properties of $\mathrm{BiFe}_{1-x} \mathrm{Mn}_{x} \mathrm{O}_{3}$," Journal of Magnetism and Magnetic Materials, vol. 310, no. 2, pp. 1177-1179, 2007.

[23] D. S. Rana, K. Takahashi, K. R. Mavani et al., "Thickness dependence of the structure and magnetization of $\mathrm{BiFeO}_{3}$ thin films on $\left(\mathrm{LaAlO}_{3}\right)_{0.3}\left(\mathrm{Sr}_{2} \mathrm{AlTaO}_{6}\right)_{0.7}$ (001) substrate," Physical Review B, vol. 75, no. 6, Article ID 060405, 2007.

[24] J. B. Goodenough, "Theory of the role of covalence in the perovskite-type manganites [La, $\mathrm{M}(\mathrm{II})] \mathrm{MnO}_{3}$," Physical Review, vol. 100, no. 2, pp. 564-573, 1955. 

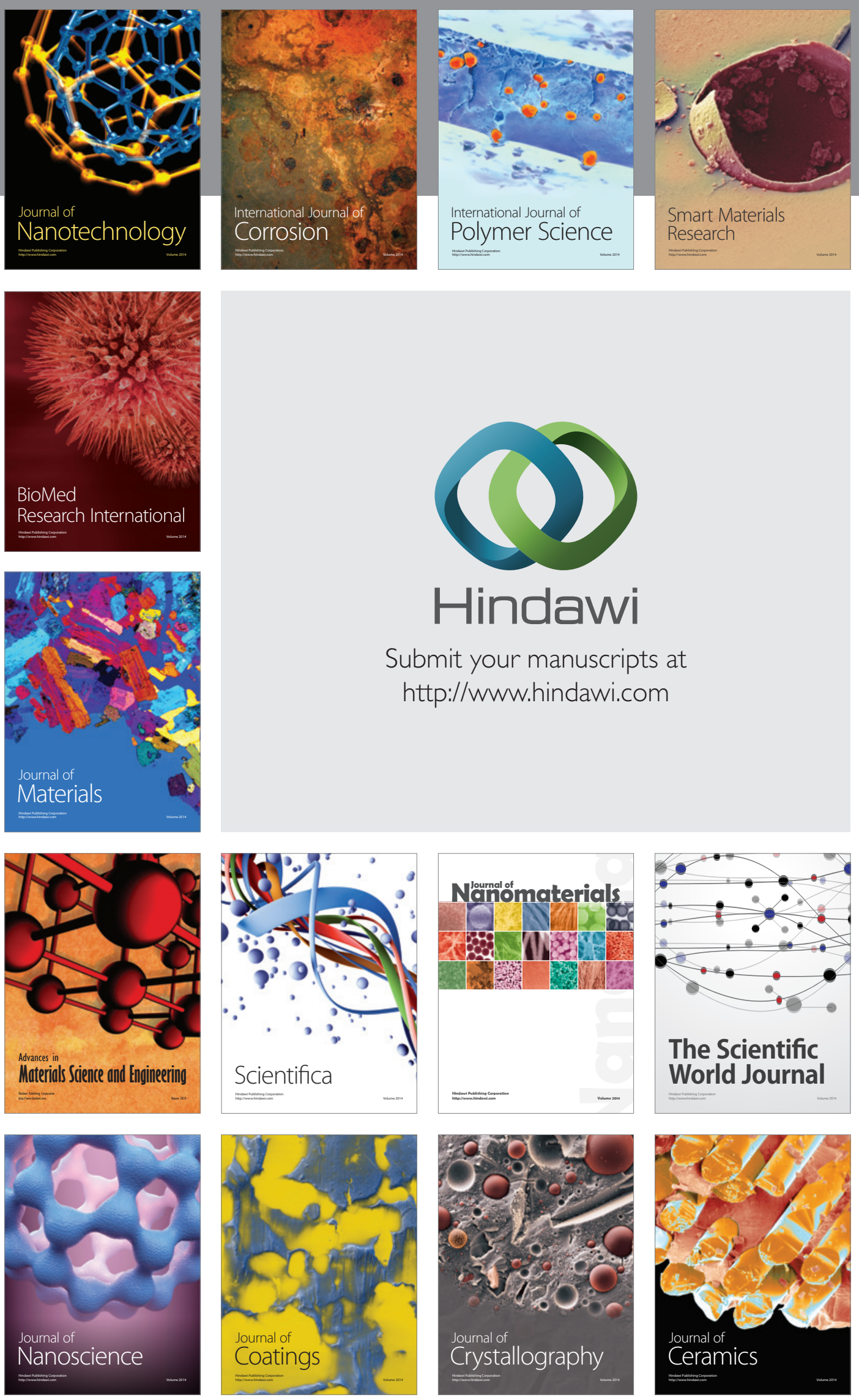

The Scientific World Journal

Submit your manuscripts at

http://www.hindawi.com

\section{World Journal}

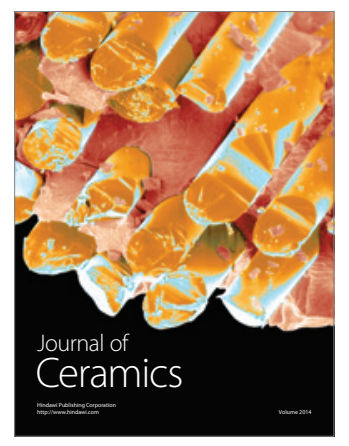

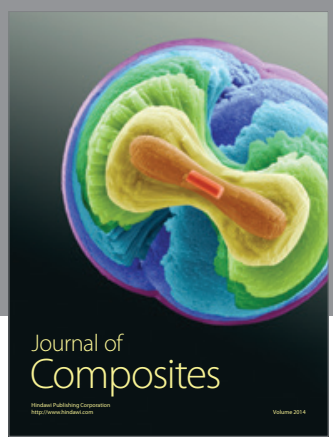
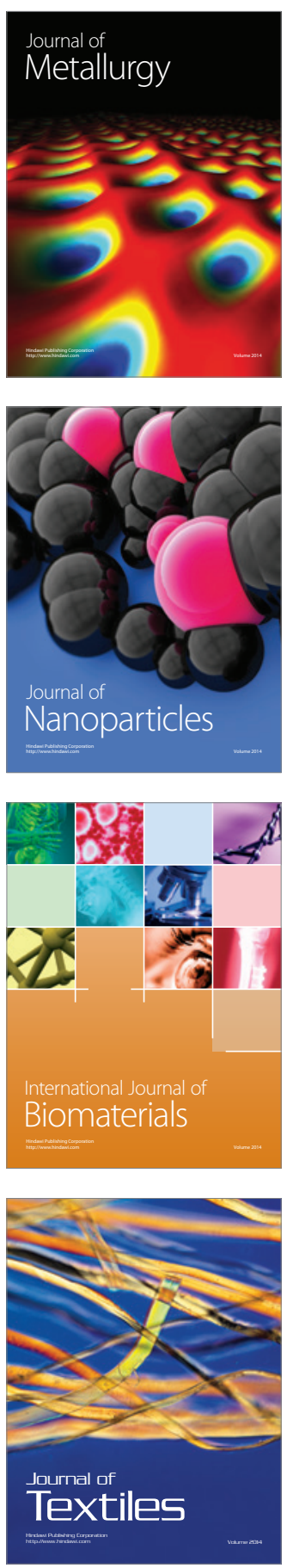